\title{
Cell coverage estimation by radio fingerprint data analytics
}

Jae-Hoon Kim ${ }^{1}$ and Woon-Young Yeo ${ }^{2^{*}}$

\begin{abstract}
We develop a novel cell coverage estimation method based on radio fingerprints collected from practical wireless service systems. A large size radio fingerprint map that shows cell identifiers and signal strength measurements on grid segmentation is built for effective estimation of cell coverage. An essential part of cell coverage estimation is radio fingerprint data cleansing and compensation. Based on this proposed iterative fingerprint data analysis method, we detect the proper cell borderline for each cell site. By the proposed method, we can efficiently estimate the cell coverage of each cell site without difficult manual field measurements. Moreover, mobile service providers can economically plan network configuration and manage subscribers using these advances in cell coverage estimation.
\end{abstract}

Keywords: Cell coverage; Radio fingerprint; Data analytics

\section{Introduction}

Accurate estimate of cell coverage is essential for cellular network design and deployment. Cell coverage is usually defined as the maximum distance, from a mobile user to a serving cell site, while maintaining sufficient service quality. It is well known that cell coverage is related to signal attenuation or path loss. The maximum path loss determines the maximum cell coverage. The OkumuraHata [1] or COST-231 [2] models are generally utilized as path loss models. The various link budget parameters shown in [3] are applied as input factors to the path loss models, then useful information can be extracted for network deployment, such as the location of cell sites required to cover the target area. Some of these parameters are within the control of the designer, such as transmit (Tx) power levels, antenna orientation, or tilt [4]. By manipulating these parameters, network operators try to optimize wireless cell coverage. The literature [5-7] describes several methods for optimizing wireless cell coverage. An optimization method focuses on coverage and antenna configuration by using a simulated annealing/tabu search [5]. A multi-objective algorithm is used to determine a series of solutions for locating the

\footnotetext{
* Correspondence: wyyeo@sejong.ac.kr

²Department of Information and Communications Engineering, Sejong University, 98 Gunja-dong Gwangjin-gu, Seoul 143-747, South Korea

Full list of author information is available at the end of the article
}

access points of wireless local area networks (WLANs) to maximize coverage and QoS [6]. A multi-criteria genetic algorithm has also been presented that selects cell sites from candidate sets with the goal of maximizing infrastructure, cost efficiency, and coverage while constraining pairwise cell overlap [7]. Another focus of research on cell coverage is the inverse relationship of coverage to traffic load in a cell. An increase in the volume of active traffic in the cell causes the interference at the cell site, and effective cell coverage is consequently decreased [8]. The coverage estimation based on traffic load estimation in a cellular network was thoroughly analyzed in [9]. They calculated the outage rate caused by interference and then restricted the effective cell coverage within an outage rate threshold.

The exact estimation of cell coverage is applicable to cellular network management. Combined with demographics and foot traffic of a particular area, decisions of cell split, addition, or re-configuration are performed. In this article, we propose a novel cell coverage estimation method using radio fingerprint data. A radio fingerprint map is built based on the measured radio fingerprint data. A grid segmentation of the fingerprint map provides an efficient frame in which to store a large amount of fingerprint data. Each grid contains a reference cell identifier in the form of a reference Pilot Number (reference PN for 3G WCDMA) or Physical Cell ID (PCI for 
4G LTE) and its signal strength measurement value. A customized data analysis method that consists of cleansing and compensation is applied to the fingerprint map. From understanding the cell shapes, outlier fingerprint data are eliminated by the iterative data cleansing method. In addition, the data compensation method can keep fingerprint data integrity and confirm the proper cell borderline for effective cell coverage estimation. This effective coverage estimation creates powerful advantages for network planning and operation. For instance, economic planning for subscriber penetration is a good example. The scheduled subscriber penetration plan has a critical impact on radio resource planning for commercial service providers.

\section{Coverage estimation by radio fingerprint maps}

The traditional method of estimating cell coverage is tracking the radio signal over the actual fields. Figure 1 shows typical ways to track radio signals. The diagnostic machine (DM) attached to a mobile station (MS) collects the radio signal while travelling a test route in Figure $1 \mathrm{a}$. Figure $1 \mathrm{~b}$ shows an example of a test route. The selected test route is usually located at the intersection of two adjacent cell sites.

Radio signal tracking is one of the most intricate processes in wireless network planning. It needs a specialized diagnostic machine and analysis procedure. The radio signal should be scanned during a specific time band and analyzed by a custom-designed tool. Furthermore, the entire procedure of tracking and analysis is performed manually. This complexity restricts the wide adoption of manual tracking, despite its relatively higher estimation precision. In [10], researchers suggest a variation of manual tracking to estimate cell coverage. A cell site could receive and determine the signal quality measurements of forward and reverse links at a particular location of a MS. The distribution of measured signal quality information could determine cell coverage.

As an alternative to manual tracking, we adopt beam lobe estimation (see Figure 2). The beam lobe estimation is based on radio propagation. The propagation shape of a radio beam can be estimated by path loss models [11]. Then, the shape of the beam lobe is calibrated considering geographical objects (e.g., buildings and roads) to estimate the actual cell coverage. However, radio propagation models contain various errors and uncertainties, such as noise level fluctuation, traffic loads, moving objects, and so on. The beam lobe model needs both of geographical map and manual measurement of cell configuration (i.e., center direction and antenna beamwidth of radiation). The $3 \mathrm{D}$ geographical map data may be obtained from an external map building company. However, the purchasing or operational cost of external maps is the problem of mobile service providers. The purchasing cost of the entire 3D digital map of South Korea is approximately one million USD and the periodic maintenance of geographical map is additionally charged. In addition, the manual measurement of cell configuration is laborious for mobile service providers. Considering the large number of base stations and cells, the manual process can contain several erroneous measurements. In the ongoing operational phase in particular, there can be many operational mistakes in measurements, which can adversely affect practical system performance.

The proposed cell coverage estimation uses the radio fingerprint map to overcome the limitations of beam lobe estimation and manual signal tracking. The radio fingerprint

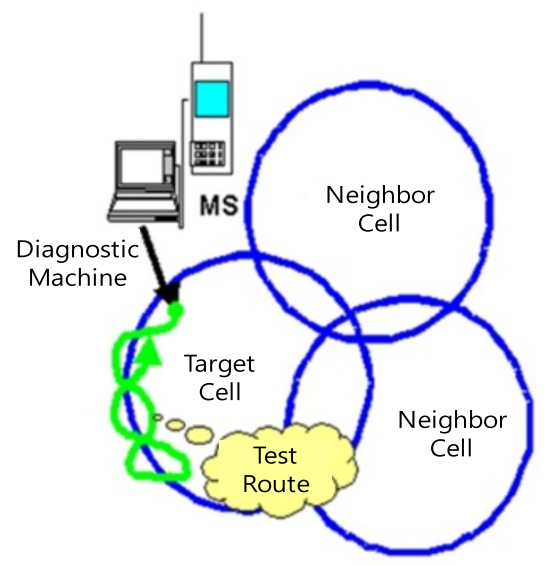

(a)

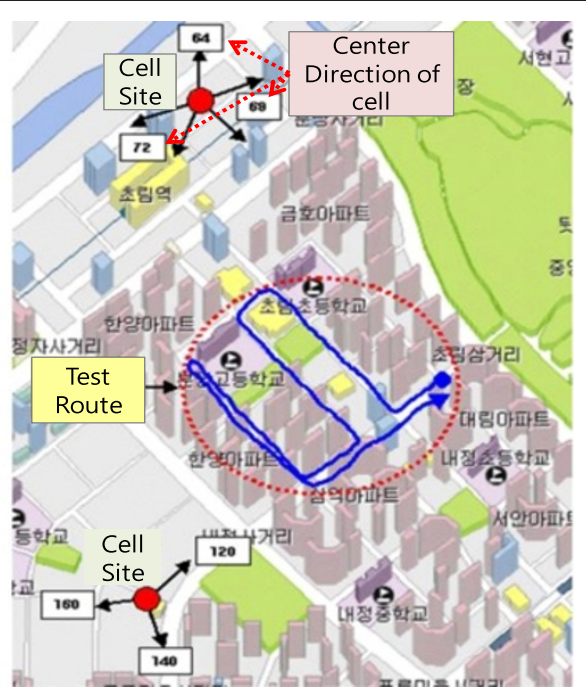

(b)

Figure 1 Radio signal diagnosis for cell coverage measurement. (a) Coverage measuring according to cell edge. (b) Route selection for measurement. 


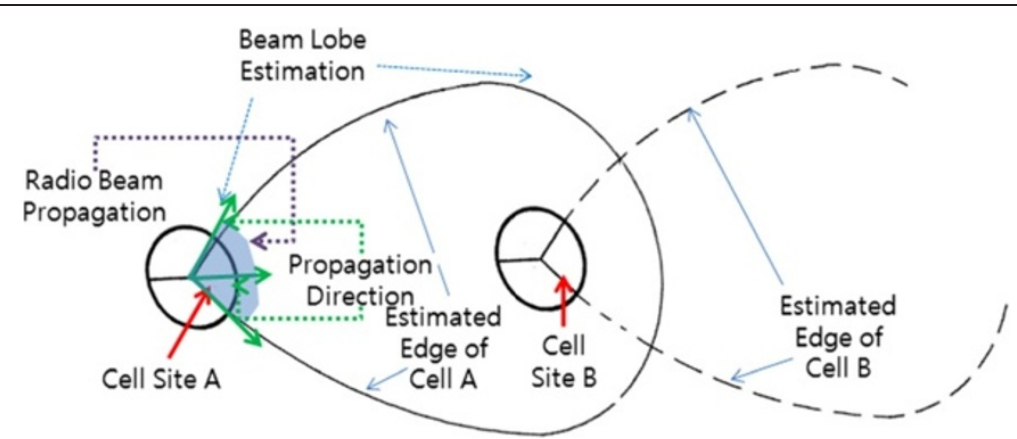

Figure 2 Beam lobe model application for cell coverage estimation.

data contains cell identifiers and signal strength indexes. The cell identifier is the reference pilot number for 3G WCDMA or physical cell ID for 4G LTE. The signal strength index denotes the Received Signal Strength Index (RSSI). In general, radio fingerprint data are collected by wardriving that is suitable for collecting fingerprint data over a wide range [12]. Data acquisition methods are based on a variety of modes of transport such as walking, bicycles, motorcycles, and cars. In small urban environments, walking or travelling by bicycle can be sufficient for data collection. However, for large-scale fingerprint data collection, fingerprint data should be collected using a fully equipped vehicle. The entire process of vehicle-based wardriving is fully automated and the radio fingerprint map is automatically generated. Figure 3 shows an example of a radio fingerprint map in a cellular system. Figure 3a shows a simplified diagram for fingerprint collection by a scanning vehicle. A scanning vehicle runs through the metropolitan area to make an entire radio fingerprint map. For efficient fingerprint collection, a fingerprint collector segments an entire area into multiple fractions and builds efficient scanning routes for each fraction. The most popular method of building scanning routes is Chinese Postman
Routing. Chinese Postman Routing is a very well-known postman tour or route inspection method of finding the shortest closed path or circuit that visits every edge of a graph. This method can be used to obtain the optimal Eulerian circuit (a closed walk that covers every edge once). The complexity to solve the Chinese Postman Routing is known as $O\left(V^{3}+E\right) . V$ denotes the intersection of roads and $E$ denotes the sectioned roads.

The radio fingerprint map in Figure $3 \mathrm{~b}$ is composed of a large number of grids. The grids have a fixed size (for example, $50 \times 50 \mathrm{~m}$ ) in a given geographical region, and each grid is indexed. Figure 4 shows the numeric cell identifier that is specified by the radio propagation from cell sites. In 3G WCDMA, the pilot number has a numeric range from 1 to 512 and the Physical Cell ID value is taken from the address space of 504 IDs for 4G LTE. The MS gets the PN or PCI from the parameters used for the downlink reference signals via a cell broadcasting channel. Given the distribution of radio fingerprints on grids, we can effectively estimate the cell coverage, as shown in Figure 4. The grid borderline of a specific cell identifier shows the specific cell coverage itself. Figure 4a represents the cell coverage over the radio fingerprint

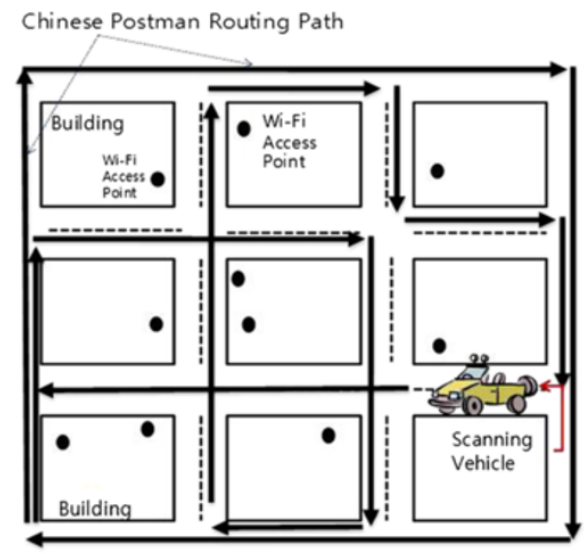

(a)

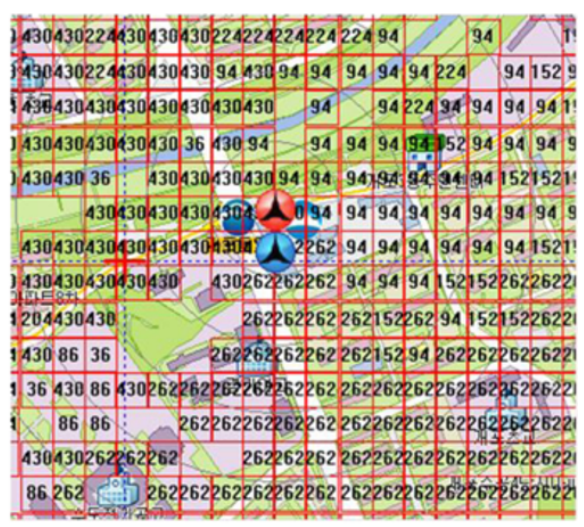

(b)

Figure 3 Example of radio fingerprint map. (a) Scanning of fingerprint. (b) Structure of fingerprint map. 


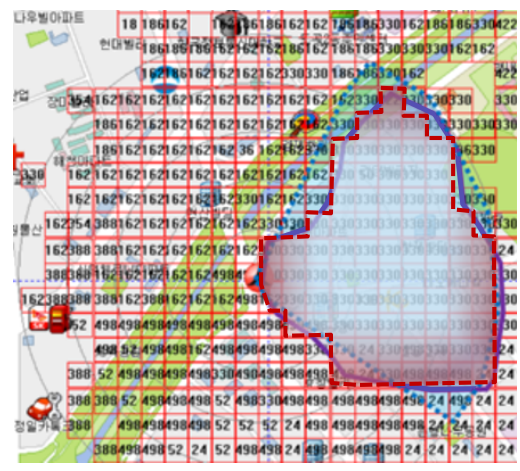

(a)

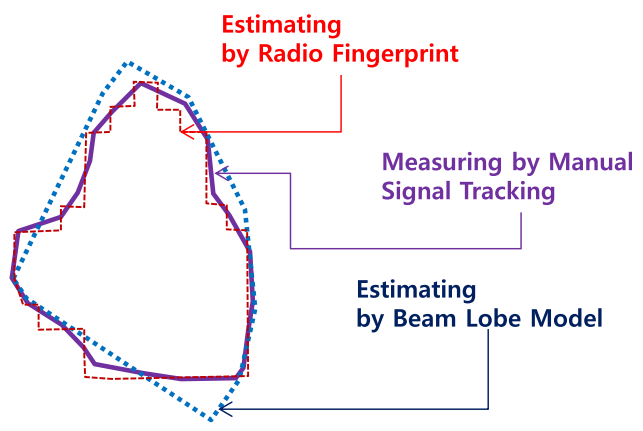

(b)

Figure 4 Coverage estimation by radio fingerprint. (a) Coverage illustration on map. (b) Coverage comparison.

map and Figure $4 \mathrm{~b}$ shows the difference between this method and other coverage estimation methods: beam lobe estimation and manual tracking.

Note that in practical cellular systems, there are many interference factors. A single grid is influenced by multiple cell sites and radio relay stations. Thus, a single grid can have multiple cell identifiers with different signal strength indexes. We should determine a single cell identifier per grid as a reference cell identifier (ref. Cell$I D)$ for fingerprint-based cell coverage estimation. The grid borderline of a ref. Cell-ID shows the coverage of cell with the ref. Cell-ID. Generally, the cell identifier with the highest RSSI becomes the ref. Cell-ID. However, the complexity of radio interference leads to errors for RSSI measurement. If the selection of cell identifiers from the highest RSSI is only a way to set the ref. CellID in all cases, we find many irregularities in the distribution pattern of a ref. Cell-ID. In addition to a typical three-sector cell formation (e.g., Figure 5a), we can find various atypical patterns (e.g., Figure 5b,c) in empirical studies. These irregularities need careful pre-processing of the radio fingerprint data for effective cell coverage estimation. Proper fingerprint data analytics such as cleansing and compensation for grid borderline detection are essential to fingerprint-based estimation of cell coverage.

\section{Radio fingerprint data analytics for grid borderline detection}

Figure 6 shows an example of cell borderline detection. Using proper fingerprint data cleansing and coherent compensation, we find the definite cell borderline. The cell borderline defines cell coverage clearly.

In Figure 6, we can find the white ref. Cell-ID numbers that are newly compensated ref. Cell-IDs. The grids with the ' $\mathrm{X}$ ' marker are grids eliminated by the cleansing procedure. The yellow line shows the final borderline of the target cell coverage. As shown in the example of cell borderline detection, the cleansing and compensation are essential analytical tools to identify the cell coverage.

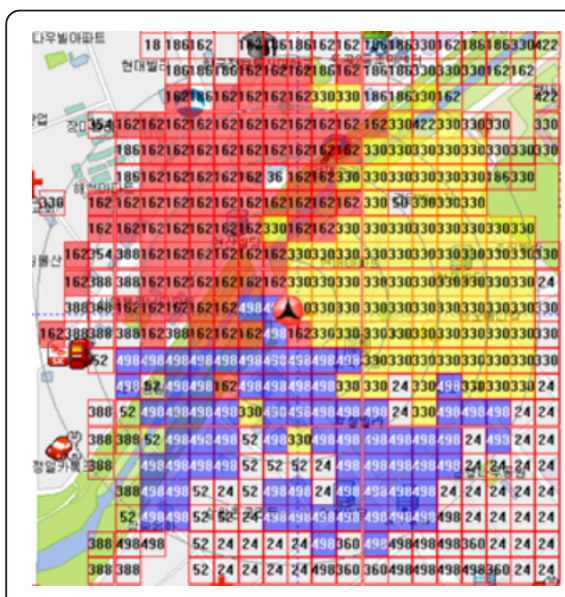

(a)

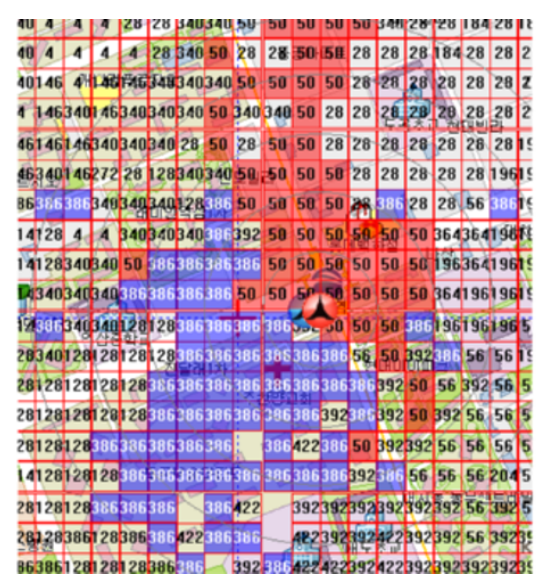

(b)

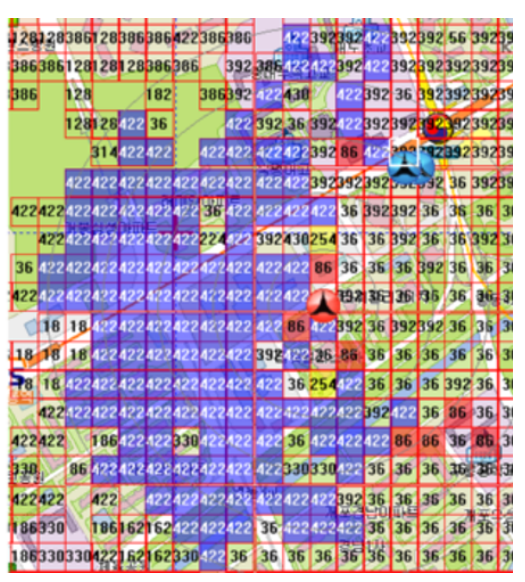

(c)

Figure 5 Various cell formations. (a) Typical three-sector cell. (b) Two-sector cell. (c) Omni cell. 


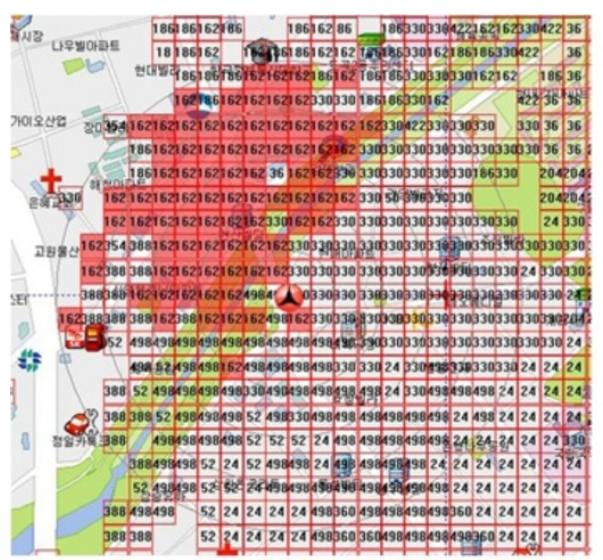

(a)

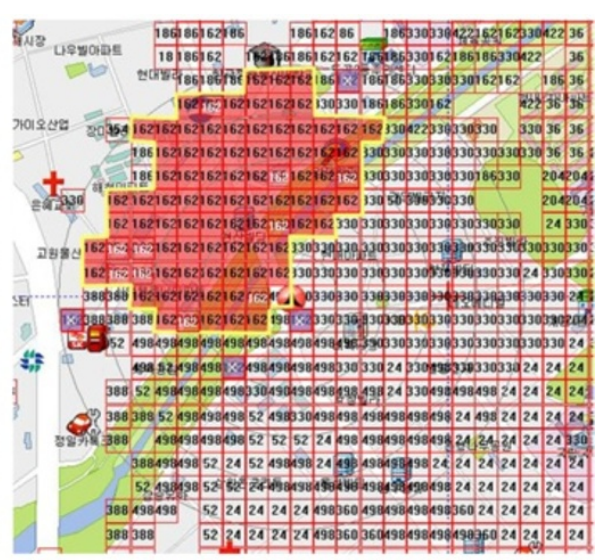

(b)

Figure 6 Example of cell borderline detection. (a) Original fingerprint.

(b) Compensated fingerprint and borderline detection.

A logical solution for many problems is to clean the data to enhance the solution quality: exploring the data set for possible problems and endeavoring to clean the errors. Of course, for any real world data set, doing this data cleansing and/or relational data integrity analysis 'by hand' is completely out of the question, given the amount of person-hours required [13]. While a data cleansing method can uncover a number of possible errors in a data set, it does not address other, more complex errors. Errors or irregularities that involve relationships among multiple fields are often very difficult to uncover. These types of errors require serious inspection and analysis to be cleaned: statistical [14], clustering [15], patternbased [16], and association [17] techniques are used to identify patterns that can uncover the data error or irregularities. In addition, informative patterns [17] and 'garbage patterns' of meaningless or mislabeled patterns [18] are used to perform data cleansing. Machine learning techniques are also used to cleanse data in the written character classification problem. However, none of the mentioned researches can present an effective tool for general data cleansing. A data cleansing activity has a very domainspecific applicability [19]. General purpose data cleansing methods, such as the Kalman filter [20], do not have the specific knowledge needed to effectively eliminate the outliers to detect the borderline of a cell. Thus, we developed a customized data cleansing method for effective fingerprint-based cell coverage estimation.

The majority of errors originate at the boundaries of cell coverage. The typical cell of a circular sector form has two types of boundaries: arc section and line section (see Figures 7 and 8). Errors in the arc section can be easily detected and fixed. The majority of errors in these sections have been found to be a form of 'island grid' identified as the red circles in Figure 7. A simple inspection of the ref. Cell-IDs of neighbor grids is sufficient to detect grids with erroneous fingerprints. The coherent compensation is then applied to these grids. The compensation process is described in the latter part of section 3. However, errors in line sections are hard to detect and fix. The black circles in Figure 7 show examples of grids in line sections. Because of the proximity to the cell center, multiple Cell-IDs with strong RSSI measurements are usually detected for each grid. Thus, it is hard to determine which grid belongs to a specific cell. To determine and fix the errors in line sections, an iterative fingerprint cleansing is proposed. This iterative cleansing method uses the phenomena of directional radio radiation that make a cell form into a circular sector. Figure 8 shows a typical three-sector cell model: a single base station supports $\alpha, \beta$, and $\gamma$ cells. Each cell has a circular sector form. A circular sector has two determinants that characterize its shape: center direction and antenna beamwidth. The antenna beamwidth can be determined by the leftmost and rightmost directions of the circular sector.

The irregular fingerprints in the line sections are detected by finding the leftmost and rightmost directions of a circular sector cell form. Given the leftmost and rightmost directions, we can detect the grids that are located outside of the target cell. When the function direction $(i)$ returns the angular direction of grid $i$, the grids that satisfy the following condition (1) are determined to be outliers.

$$
\left\{\begin{array}{l}
\operatorname{direction}(i)<d_{\text {right }} \text { or } \operatorname{direction}(i)>d_{\text {left }}, \text { if } d_{\text {left }}>d_{\text {right }} \\
\text { direction }(i)<d_{\text {right }} \& \operatorname{direction}(i)>d_{\text {left }}, \text { if } d_{\text {left }}<d_{\text {right }}
\end{array}\right.
$$

where $d_{\text {left }}$ is the leftmost direction of a circular sector cell form and $d_{\text {right }}$ is the rightmost one. The following pseudocode details the process of finding erroneous 


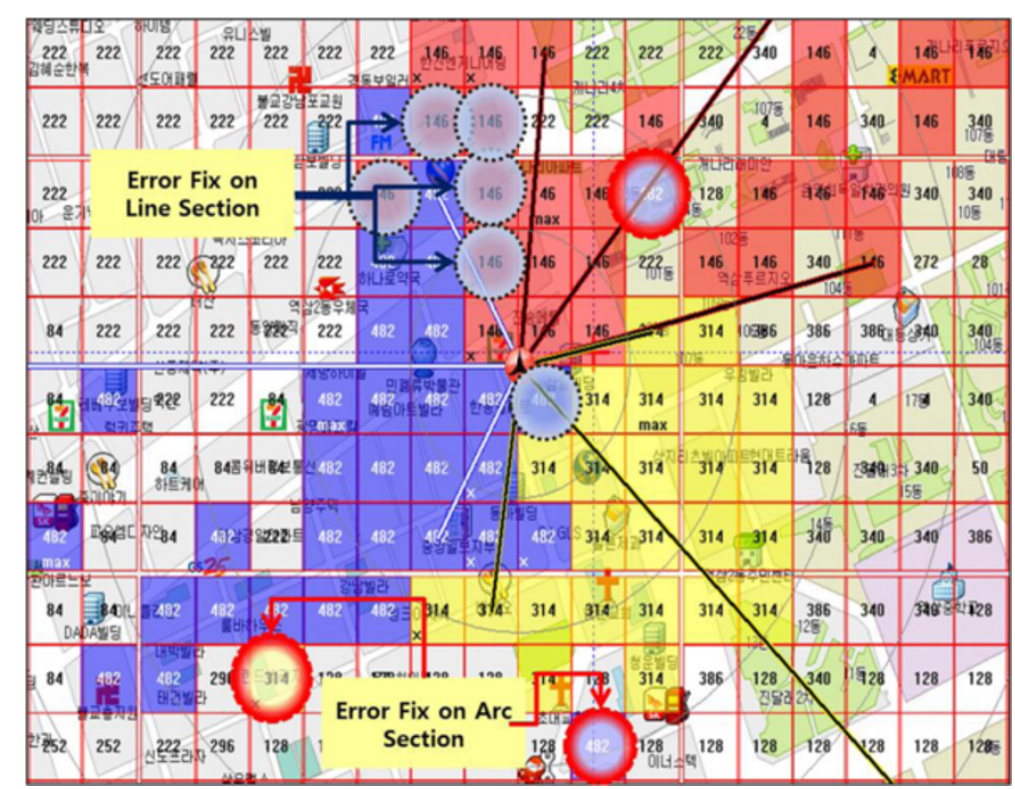

Figure 7 Errors in arc/line section.

fingerprint data given the leftmost and rightmost directions. Note that the following code also includes erroneous fingerprint detection in the arc section by a simple inspection of the ref. Cell-IDs of neighbor grids.

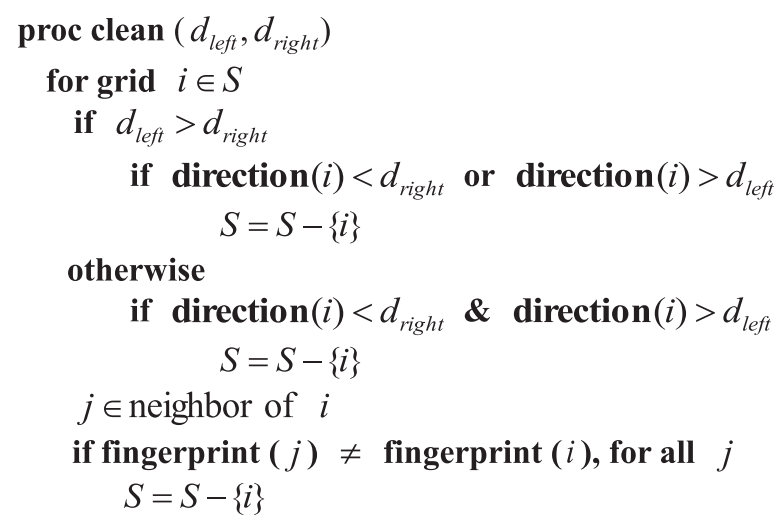

The $S$ means a set of grids which have the same ref. Cell-ID. The values of $d_{\text {left }}$ and $d_{\text {right }}$ of the target cell are updated during the iterative calculation of antenna beamwidth and center direction of the target cell. After the fingerprint data cleansing is performed by the above code using the current $d_{\text {left }}$ and $d_{\text {right, }}$, we obtain newly updated proper fingerprint data (i.e., the updated ref. Cell-ID data for each grid). Using the updated proper ref. Cell-ID data, we calculate the updated antenna beamwidth for each cell. The antenna beamwidth of a target cell can be calculated by counting the number of grids $\left(g_{c}\right)$ that have the target cell's ref. Cell-ID within the current $d_{\text {left }}$ and $d_{\text {right. }}$ The counting procedure is described in the following pseudocode.

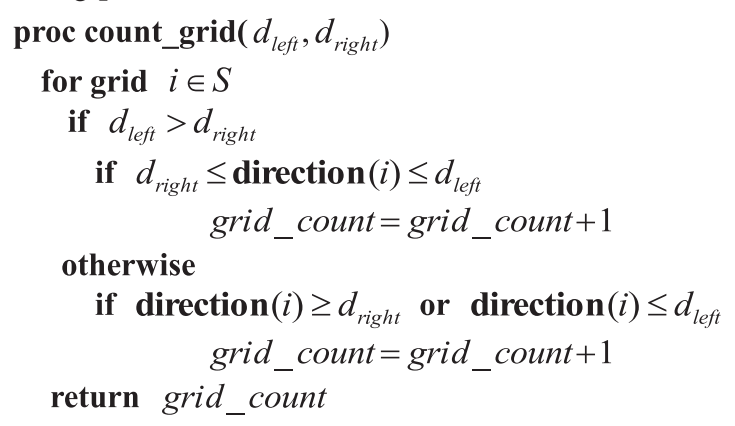

The antenna beamwidth of the $n$th iteration $\left(w_{c}^{n}\right)$ can now be given as

$$
w_{c}^{n}=360 \times\left(g_{c} / g_{\mathrm{BS}}\right)
$$

where $g_{\mathrm{BS}}$ is the total number of grids in the analysis area. A series of circular areas from the center of the cell can be selected as the analysis area. For example, circles with a 100-, 200-, or 500-m radius can be the target analysis area. The analysis area does not have any relation to cell coverage itself. But, we obtain the antenna beamwidth by grid counting and the ratio of $g_{c}$ over $g_{\mathrm{BS}}$. Thus, we should set a standard circular area to obtain proper ratio of $g_{c}$ over $g_{\mathrm{BS}}$. The larger area contains a larger number of grids for counting and gives sufficient ref. Cell-ID data to estimate the borderlines. However, a 


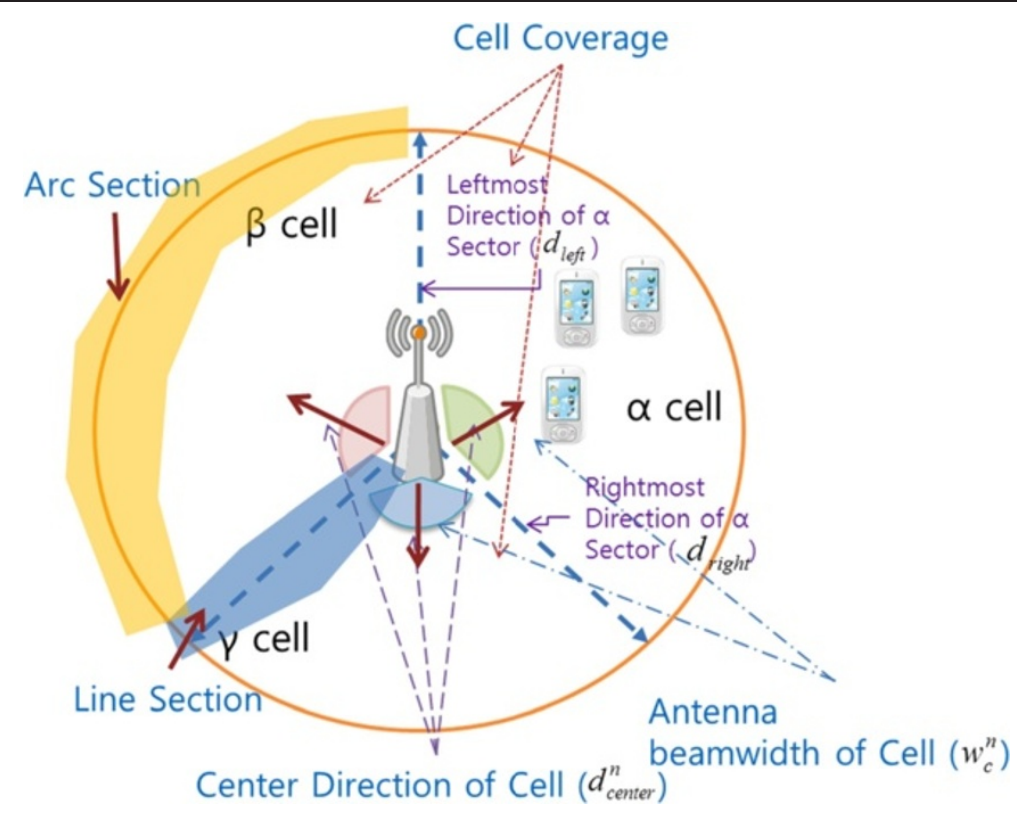

Figure 8 Diagram of a typical three-sector cell model.

larger area contains many erroneous ref. Cell-IDs which would be invalid for borderline detection.

The center direction of the target cell is simply estimated using the current $d_{\text {left }}$ and $d_{\text {right }}$. We can obtain the center direction of the $n$th iteration $\left(d_{\text {center }}^{n}\right)$ by simply aligning the center of the boundary grids as follows (3).

$$
\begin{cases}d_{\text {center }}^{n}=d_{\text {right }}+\frac{1}{2}\left(d_{\text {left }}-d_{\text {right }}\right), & \text { for } d_{\text {left }}>d_{\text {right }} \\ d_{\text {center }}^{n}=d_{\text {right }}+\frac{1}{2}\left(\left(360-d_{\text {right }}\right)+d_{\text {left }}\right), & \text { for } d_{\text {left }}<d_{\text {right }}\end{cases}
$$

After fixing the antenna beamwidth and center direction of the target cell for the $n$th iteration, we recalculate the leftmost and rightmost directions of the target cell as follows (4):

$$
d_{\text {left }}=d_{\text {center }}^{n}+\frac{1}{2} w_{c}^{n}, \quad d_{\text {right }}=d_{\text {center }}^{n}-\frac{1}{2} w_{c}^{n}
$$

Then, the cleansing procedure runs again with the newly updated $d_{\text {left }}$ and $d_{\text {right }}$. The new cleansing procedure determines the center direction and antenna beamwidth of $n+$ 1 th stage. This iterative approach continuously enhances the accuracy of the cell borderline detection in the line section. The iteration finishes when the difference between the $n$th and $n-1$ th stage values is within a certain bound (i.e., $\left|w_{c}^{n}-w_{c}^{n-1}\right| \leq \delta$ ). The following pseudocode presents the entire iterative structure for cleansing fingerprint data.

let analysis area set, area $=\{100,200,300,400,500\}$

let grid $i \in S$ for all $i$

measure leftmost direction of target cell, $d_{\text {left }}$

measure rightmost direction of target cell, $d_{\text {right }}$

let grid $i \in S$ for fingerprint $(i)=$ ref.Cell-ID (target cell)

while $\left(\left|w_{c}^{n}-w_{c}^{n-1}\right| \leq \delta\right)$

clean $\left(d_{\text {left }}, d_{\text {right }}\right)$

if $d_{\text {left }}>d_{\text {right }}$ center direction of cell, $d_{\text {center }}^{n}=d_{\text {right }}+\frac{1}{2}\left(d_{\text {left }}-d_{\text {right }}\right)$

otherwise center direction of cell, $d_{\text {center }}^{n}=d_{\text {right }}+\frac{1}{2}\left(\left(360-d_{\text {right }}\right)+d_{\text {left }}\right)$ if $d_{\text {center }}^{n}>360$

$$
d_{\text {center }}^{n}=d_{\text {center }}^{n}-360
$$

$g_{c}=$ count_grid $\left(d_{\text {leff }}, d_{\text {right }}\right)$

$g_{B S}=$ total number of grids in analyzing area

let beamwidth, $w_{c}^{n}=360 \times\left(g_{c} / g_{B S}\right)$

$$
\begin{aligned}
& d_{\text {left }}=d_{\text {center }}^{n}+\frac{1}{2} w_{c}^{n} \\
& d_{\text {right }}=d_{\text {center }}^{n}-\frac{1}{2} w_{c}^{n} \\
& n=n+1
\end{aligned}
$$

The computational complexity of the aforementioned iterative method depends completely on the $\delta$ and the 


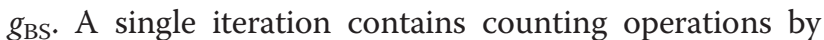
$g_{\mathrm{BS}}$ times. When the difference bound $(\delta)$ is given, the number of iterations is limited to $360 / \delta$ times. Therefore, the overall complexity of cleansing for a single cell is bounded as $O\left(360 / \delta \times g_{\mathrm{BS}}\right)$. In the case of the difference bound $(\delta)$ as the smallest integer value (i.e., $\delta=1$ ), where all angular directions are measured by an integer value, the number of iterations is limited to 360 times. Therefore, the overall complexity of cleansing for a single cell is practically bounded as $O\left(360 g_{\mathrm{BS}}\right)$.

Next, we perform a coherent compensation process to assign the proper ref. Cell-IDs to erroneous grids in the arc section. We can calibrate the imperfectly measured RSSI values in the radio fingerprint by using the compensated ones. The imperfection of measurement is observed as the temporal fluctuation of RSSI measurement. The temporal fluctuation of RSSI measurements are widely observed in collecting and building a radio fingerprint map. Figure 9 shows the concept of compensation. If an islandtype erroneous grid is detected by the inspection of the neighbor grids' ref. Cell-IDs, we select the vertical and horizontal neighbor grids and apply a smoothing technique. By curve fitting with neighboring RSSI values, the two newly compensated RSSI values are obtained according to both the vertical and horizontal axes. The final compensated RSSI value is calculated by averaging the two vertical and horizontal compensated RSSI values. When the compensation for RSSI is completed, we select the cell identifier with the highest RSSI as the new ref. Cell-ID. Note that, the temporal fluctuation of RSSI used in the proposed compensation method is the possible key to discrimination between deep-faded grids and erroneous grids. The deep-faded grids are not influenced by the compensation. The compensation method is only applicable to the temporal fluctuation of RSSI.

\section{Numerical results}

To test the proposed method, we developed a cell coverage estimation program which has a map of an urban (Gangnam) area in South Korea. The test area has a total of 47,439 grids and we collected complete ref. Cell-ID and RSSI (i.e., fingerprint) data by actual wardriving. The cell coverage estimation program includes all the collected fingerprint data of the grids and the position data of cell sites. The positions of cell sites were obtained using an information database from a commercial WCDMA system. In addition, the cell coverage estimation program contains the position information of the relay stations. All the information for grids, cell sites, and relay stations are shown in a map and information window of the cell coverage estimation program. The area shown in the map window can be moved by simple mouse drag operations. The function of map panning in/out is implemented by simple menu directions.

To determine the coverage of the cell site, we first simply select a cell site in the map window. After the selection, we set the analysis area for the coverage. Five different radii for the analysis area can be selected: 100, 200, 300, 400, and $500 \mathrm{~m}$.

The results of the estimation are given by the iterative approach. The 'iteration' command can be activated by one button press in the coverage estimation program, giving the result of a single iteration. Each iteration is given the information of the cell areas: leftmost/rightmost directions, denoted as 'min/max degree', center direction of a cell, denoted as 'center', and antenna beamwidth, denoted

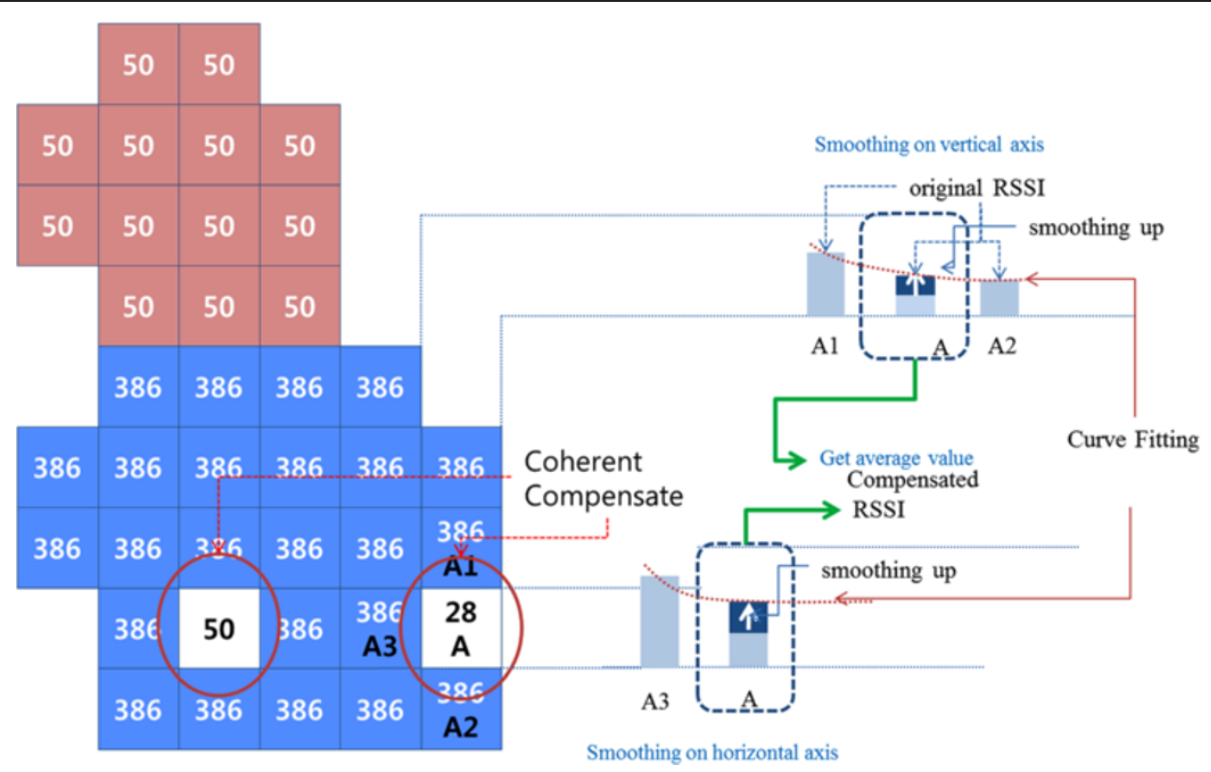

Figure 9 Coherent compensation. 
as 'width.' After performing consequent iterations, we can observe the convergence of the results. The actual convergence of center direction or antenna beamwidth are accomplished within small number of iteration, such as $5 \sim 8$ iterations for almost all cases. Figure 10 shows a screenshot of the developed estimation program.

To show the performance of the proposed coverage estimation, we define two performance criteria: conformity ratio and inclusion ratio.

In Figure 11, the areas enveloped by the solid line represent the manually measured coverage of the cell site. The area enveloped by dotted lines show the estimated coverage by the empirical models, such as beam lobe estimation or fingerprint-based estimations. From the geographical relationships among them, we classify three regions as following:

- Region $A$ : included in both the Measured and Estimated Coverage

- Region $B$ : included in the Estimated Coverage, but not in the Measured Coverage

- Region $C$ : included in the Measured Coverage, but not in the Estimated Coverage

The primary criterion is Inclusion Ratio, defined as $A /(A+C)$. A good estimation should contain a large amount of measured coverage within the estimated coverage. However, even a simple overestimation of the cell coverage can achieve a high inclusion ratio (i.e., small region $C$ ) but may include significant redundant space (i.e., large region $B$ ). The Conformity Ratio, defined as $A /(A+B)$, avoids this weakness. A higher conformity ratio guarantees a tight estimation on the measured coverage (i.e., small region $B$ ). Therefore, we can evaluate the quality of the estimation using these two numerical performance criteria.

Figure 12 shows 15 different cases for the WCDMA cell sites. For each cell site, we calculated the inclusion and conformity ratios for fingerprint-based cell coverage estimation. The cell coverage measurements from manual signal tracking are the given as a ground truth for the inclusion and conformity ratio calculations. For comparison, the results of the beam lobe model estimation are also presented, based on the approach of a spatial moving least squares (MLS) method [21]. The improvement of the inclusion ratio is observed for all of the experiments. The average value of the beam lobe model is $85.9 \%$, and the fingerprint-based estimation is $91.4 \%$, giving an improvement of $5.5 \%$. In addition, the proposed fingerprint-based estimation also improved the conformity ratio. The average value of the beam lobe model is $85.1 \%$ and the fingerprint-based estimation $90.3 \%$, giving an improvement of $5.2 \%$. Note that, we add the fingerprint-based estimation without data cleansing and compensation. There is no significant enhancement without data analytics: slightly lower performance is

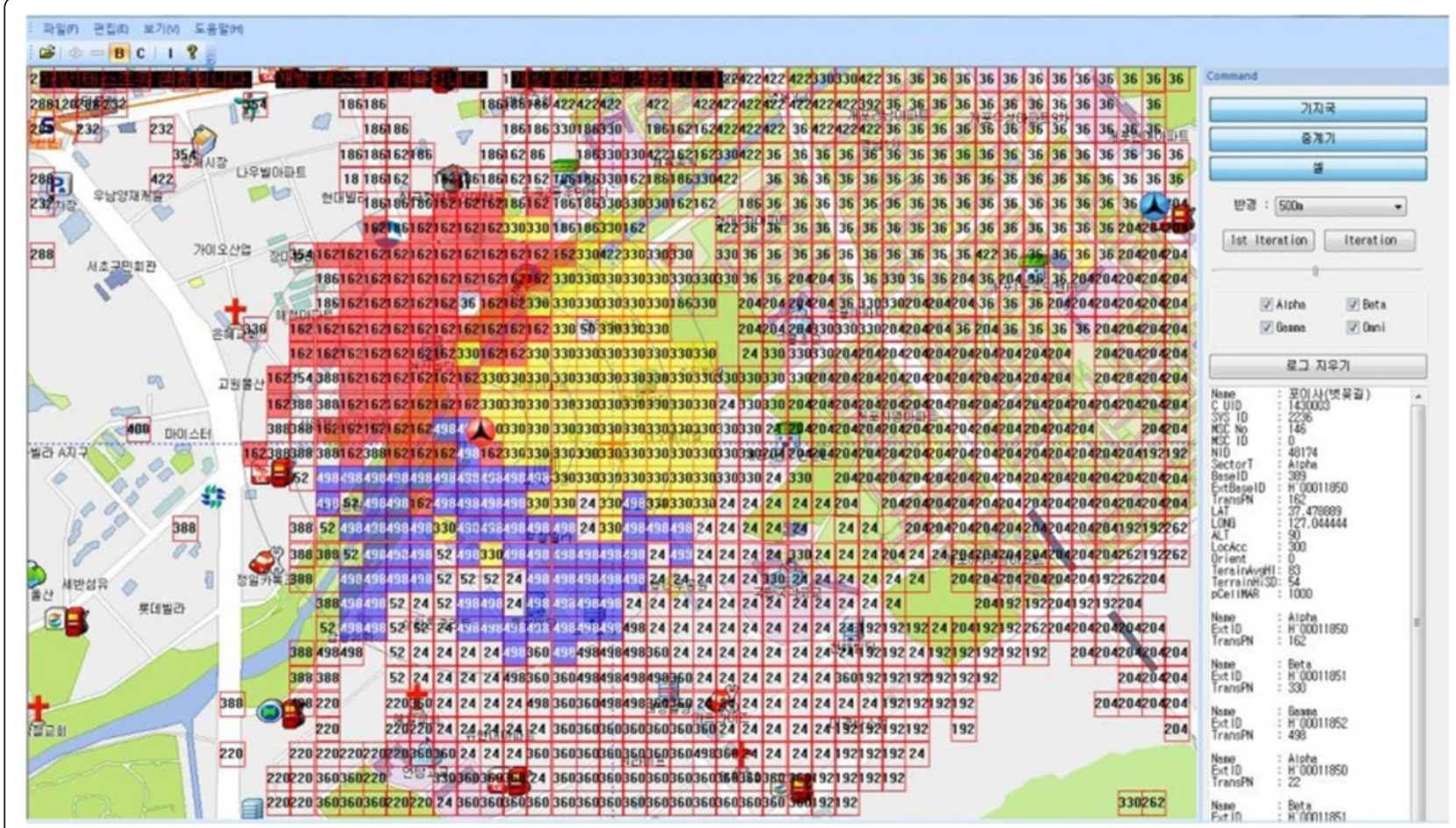

Figure 10 Screenshot of the estimation program. 


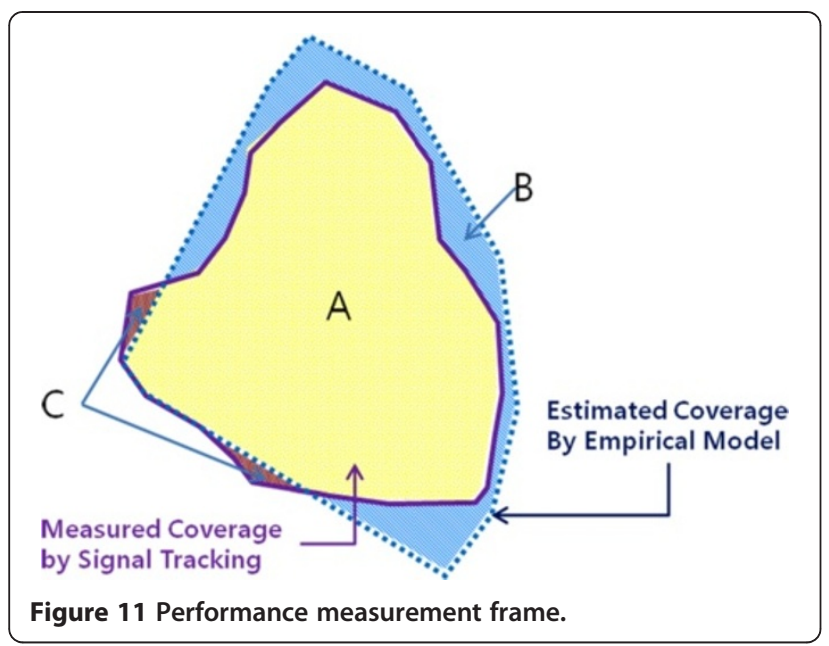

observed $(-0.6 \%$ for inclusion ratio, $-0.8 \%$ for conformity ratio).

The practical processing time of proposed cell borderline detection method is very fast. All operations of method are elementary integer arithmetic and numbers of iterations are bounded to practically less than 10 times. For the tested 15 cells, total practical processing time is less than 1 second using a plain PC server. Compared to beam lobe model estimation, which needs the logarithm calculation of path loss model, the processing time of proposed method has competitive advantage. Apparently, fingerprint collection by wardriving is a timeconsuming procedure. However, the beam lobe model also requires geographical map data for estimation.

We next extended our cell coverage estimation method to a large area. We selected districts in Gangnam, Seoul as our targets. A total of ten test districts were selected for the extended test (see Figure 13). All fingerprint data of

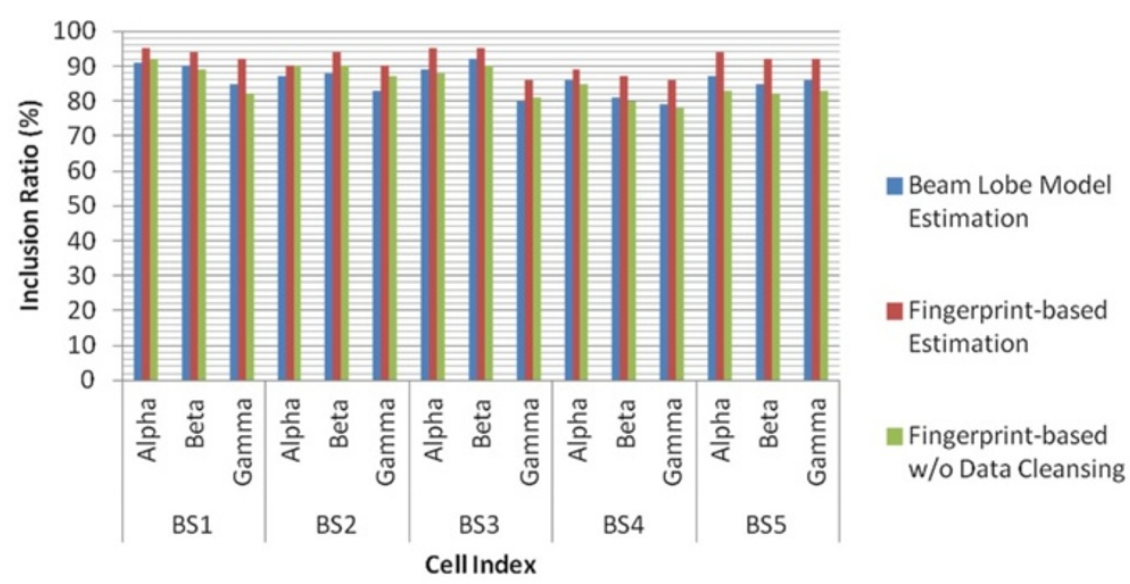

(a)

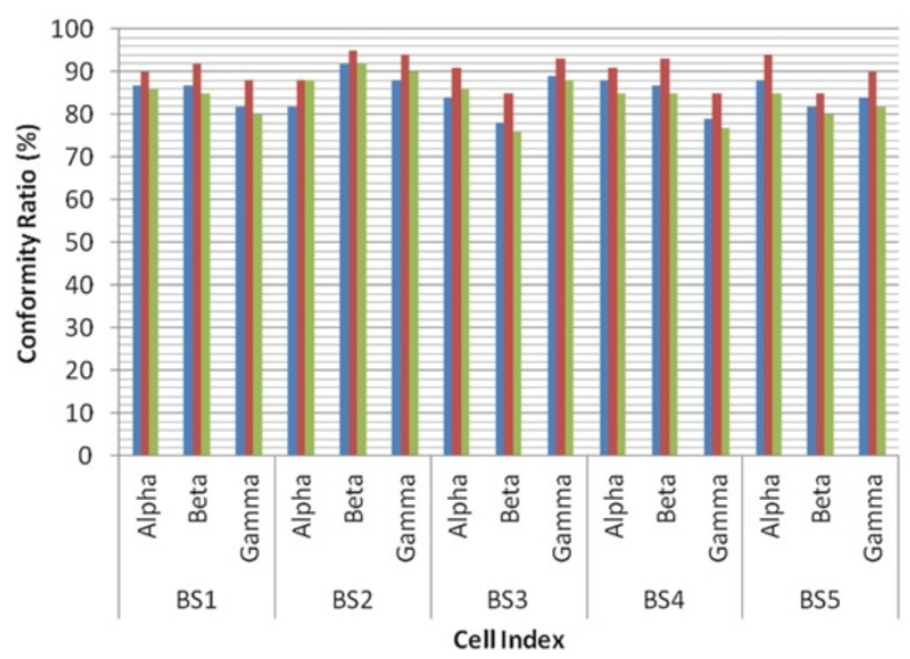
Beam Lobe Model
Estimation

Fingerprint-based Estimation

Fingerprint-based w/o Data Cleansing

(b)

Figure 12 Performance evaluation for sample cell sites. (a) Inclusion ratio. (b) Conformity ratio. 


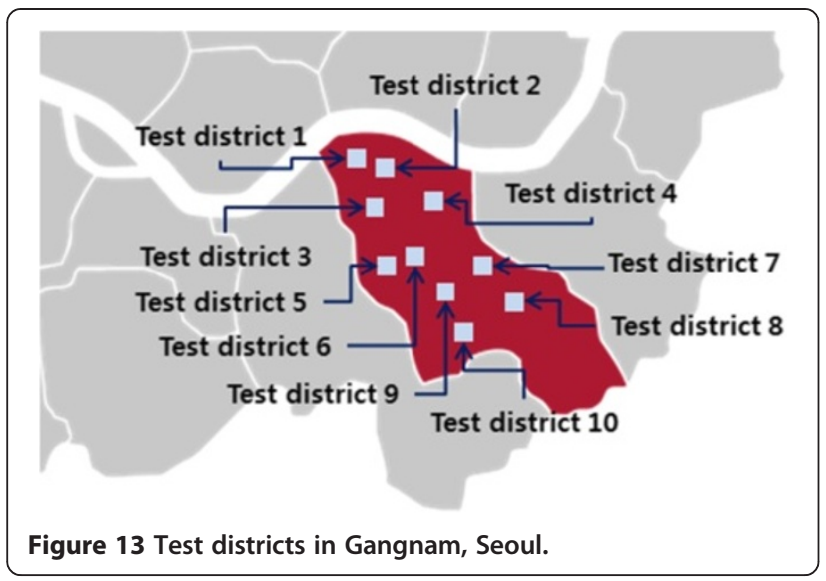

selected areas are collected by actual wardriving. Note that, the area of the Gangnam district is $39.55 \mathrm{~km}^{2}$. The cell coverage estimation method was applied at 30 cell sites for each district. The total number of tested cells in Gangnam is 300, which is a sufficient sample size to show the effects of the proposed method in the entire area. For statistical analysis, a sample size of 300 to 400 cells is suitable. See the Appendix for details of the statistical conjecture.

Figure 14 shows the estimation results which prove the effectiveness of the proposed method in the diverse environments of an urban area. An improvement of the inclusion ratio is observed for all test districts. The average value of the beam lobe model is $82.2 \%$, and the fingerprintbased estimation is $89.9 \%$, giving an improvement of $7.7 \%$.

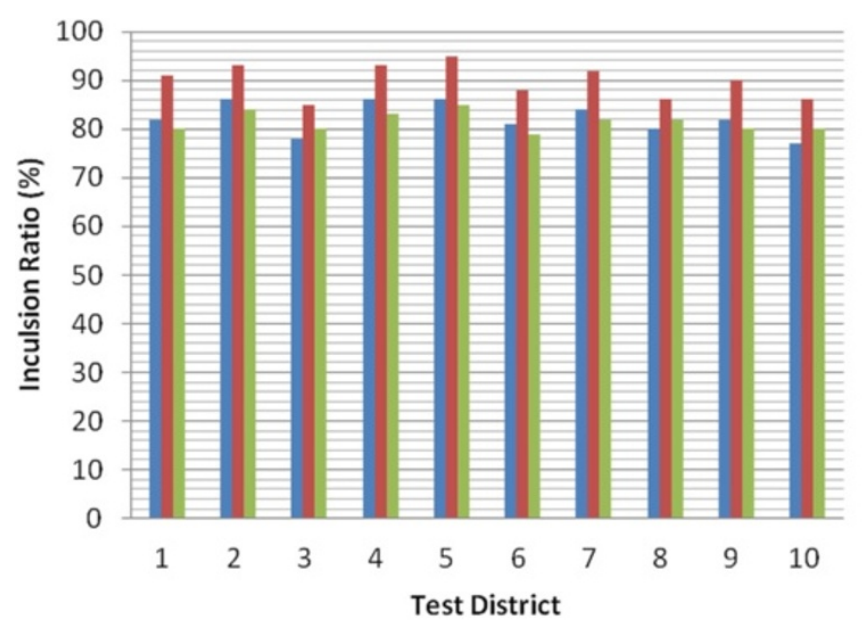
Beam Lobe Model
Estimation

- Fingerprint-based Estimation

Fingerprint-based w/o Data Cleansing

(a)

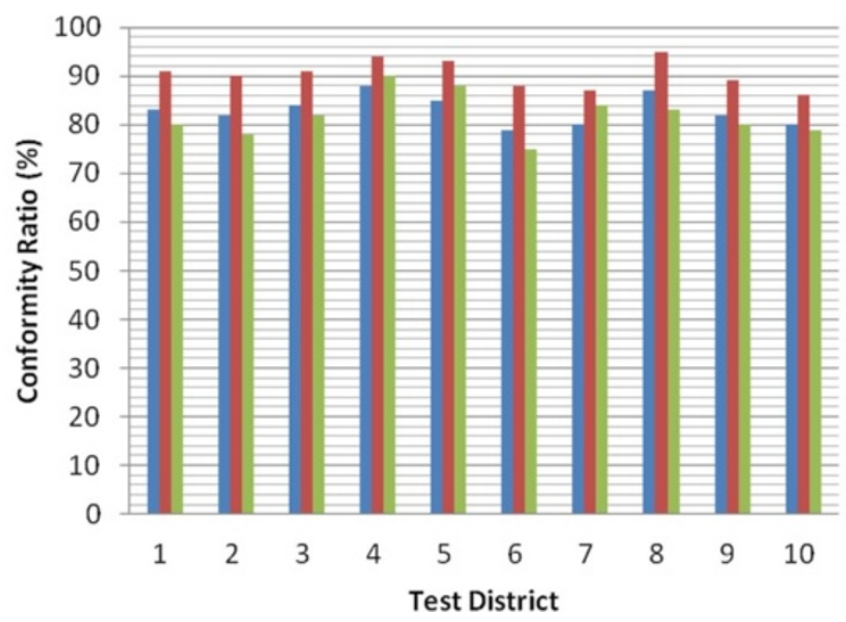

- Beam Lobe Model Estimation

- Fingerprint-based Estimtion

- Fingerprint-based w/o Data Cleansing

(b)

Figure 14 Performance evaluation for sample cell sites. (a) Inclusion ratio. (b) Conformity ratio. 
In addition, the proposed fingerprint-based estimation also improved the conformity ratio. The average value of the beam lobe model is $83.0 \%$, and the fingerprint-based estimation is $90.4 \%$, giving an improvement of $7.4 \%$. Note that, we add the fingerprint-based estimation without data cleansing and compensation. There is no significant enhancement without data analytics: slightly lower performance is observed $(-0.7 \%$ for inclusion ratio, $-1.1 \%$ for conformity ration).

\section{Conclusions}

In this paper, we dealt with cell coverage estimation. The problem is to find precise cell coverage information for proper network design and operation. By gathering complete radio fingerprint data in the test area, we make a grid map with reference cell identifiers and signal strengths. Based on this fingerprint map, we can estimate the cell coverage. The fingerprint data cleansing and compensation for borderline detection is an essential part of cell coverage estimation. Given our understanding of circular sector cell configurations, we developed an iterative data cleansing and compensation method. As a result of an appropriate cleansing and compensation method, we can detect a tight cell borderline. We presented the entire estimation procedure and a coverage estimation program that consists of a large amount of radio fingerprint data and a proposed procedure. The usefulness of the developed algorithmic procedure and coverage estimation program is proven by actual fingerprint data collected from a commercial wireless service provided by SK Telecom, Korea. A field engineer can now estimate cell coverage without manual operations using this simple coverage estimation program. Note that, the fine grid granularity guarantee higher performance. But it also generates higher cost for fingerprint data collection. Thus, we select singlesized grids in building a radio fingerprint map (e.g. $50 \times 50$ ). To enhance the accuracy on estimation, we can adopt variable granularity: fine granularity for cell border area and coarse granularity for cell inner area.

\section{Appendix}

The sample proportion $(\hat{p})$ is a good candidate for a statistical estimator. The sample proportion represents the population proportion $(p)$. The size of sample reference points $(n)$ is determined by the error tolerance limit $(\varepsilon)$. To restrict the estimation error (i.e., $\hat{p}-p$ ) within $\pm \varepsilon$, the size of the sample reference points is given as Equation 5 .

$$
n=\frac{p(1-p) Z_{a / 2}^{2}}{\varepsilon^{2}}
$$

where $Z_{a / 2}$ is the standard score (or $Z$ value) obtained from the probability table of standard normal distribution. When we set $1-a$ as the existing probability of estimation error within the error tolerance limit (i.e., $P(-\varepsilon<\hat{p}-p<+\varepsilon)=1-a$ ), $P(-\varepsilon<\hat{p}-p<+\varepsilon)$ can be transformed into $P\left(\frac{-\varepsilon}{\sqrt{p(1-p) / n}}<\right.$ $\left.\frac{\hat{p}-p}{\sqrt{p(1-p) / n}}<\frac{+\varepsilon}{\sqrt{p(1-p) / n}}\right)$. The random variable, $\frac{\hat{p}-p}{\sqrt{p(1-p) / n}}$, tends to follow a standard normal distribution. Then, $\frac{\varepsilon}{\sqrt{p(1-p) / n}}=Z_{a / 2}$. However, the population proportion $(p)$ is generally unknown. Thus, we set $p$ to 0.5 for conservative error estimation ( $\varepsilon$ is maximized when $p$ is 0.5 ). Finally, we derive the size of the sample reference points as follows:

$$
n=0.25 Z_{a / 2}^{2} / \varepsilon^{2}
$$

To restrict the estimation error (i.e., $\hat{p}-p$ ) within \pm 0.05 (i.e., the error tolerance limit is 5\%) under the $95 \%$ probability (i.e., $P(-0.05<\hat{p}-p<+0.05)=0.95)$, we select approximately 384 samples to represent the total population.

\section{Competing interests}

The authors declare that they have no competing interests.

\section{Acknowledgements}

This research work is supported by SK Telecom, South Korea. All data are collected using the facility of SK Telecom. This work was also supported by the National Research Foundation of Korea (NRF) grant funded by the Korean Government (2011-0011825).

\section{Author details}

'Department of Industrial Engineering, Ajou University, San5

Woncheon-dong Yeongtong-gu, Suwon 443-749, South Korea. ${ }^{2}$ Department of Information and Communications Engineering, Sejong University, 98 Gunja-dong Gwangjin-gu, Seoul 143-747, South Korea.

\section{Received: 15 January 2014 Accepted: 14 April 2014}

Published: 4 May 2014

\section{References}

1. A Medeisis, A Kajackas, On the use of the universal Okumura-Hata propagation prediction model in rural areas, In IEEE 51st Vehicular Technology Conference Proceedings, VTC 2000 Spring (IEEE, Piscataway, 2000)

2. R Mardeni, TS Priya, Optimised COST-231 Hata models for WiMAX path loss prediction in suburban and open urban environments. Mod. Appl. Sci. $4,9(2010)$

3. H Holma, A Toskala, LTE for UMTS (Wiley, New York, 2011)

4. D Fagen, PA Vicharelli, J Weitzen, Automated wireless coverage optimization with controlled overlab. IEEE Trans. VT 57, 4 (2008)

5. I Siomina, P Varbrand, D Yuan, Automated optimization of service coverage and base station antenna configuration in UMTS networks. IEEE Wireless Commun. 13(6), 16-25 (2006)

6. K Jaffres-Runser, J-M Gorce, S Ubeda, QoS-constrained wireless LAN optimization within a multiobjective framework. IEEE Wireless Commun. 13(6), 26-33 (2006)

7. R Whitaker, L Raisanen, S Hurley, The infrastructure efficiency of cellular wireless networks. Comput. Netw. 48(6), 941-959 (2005)

8. H Holma, A Toskala, WCDMA for UMTS (Wiley, New York, 2000)

9. H Jiang, CH Davis, Cell-coverage estimation based on duration outage criterion for CDMA cellular systems. IEEE Trans. VT 52, 4 (2003)

10. Lucent Inc, System for Determining Wireless Coverage Using Location Information for a Wireless Unit. US Patent US6,522,888. B1, 18 Feb 2003

11. VS Abhayawardhana, IJ Wassel, D Crosby, MP Sellars, MG Brown, Comparison of empirical propagation path loss models for fixed wireless access systems, in IEEE 61st Vehicular Technology Conference, VTC 2005-Spring (IEEE, Piscataway, 2005) 
12. H Yoshida, S Ito, N Kawaguchi, Evaluation for pre-acquisition methods for position estimation system using wireless LAN, in Proceedings of the Third International Conference on Mobile Computing and Ubiquitous Networking (ICMU 2006) (IEEE, Piscataway, London, UK, 2006), pp. 148-155

13. O Maimon, L Rokach, Data Mining and Knowledge Discovery Handbook (Springer, New York, 2005)

14. RA Johnson, DW Wichern, Applied Multivariate Statistical Analysis, 4th edn. (Prentice Hall, Upper Saddle River, 1998)

15. EM Knorr, RT Ng, A unified notion of outliers: properties and computation, in Proceedings of KDD 97 (MIT Press/AAAl Press, Cambridge, 1997), pp. 219-222

16. L Kaufman, PJ Rousseauw, Finding Groups in Data: An Introduction to Cluster Analysis (Wiley, New York, 1990)

17. I Guyon, N Matic, V Vapnik, Discovering Information Patterns and Data Cleaning, in Advances in Knowledge Discovery and Data Mining, ed. by UM Fayyad, G Piatetsky-Shapiro, P Smyth, R Uthurasamy (MIT Press/AAAI Press, Cambridge, 1996), pp. 181-203

18. CJ Date, An Introduction to Database Systems (Addison-Wesley, Reading, 1990)

19. ML Lee, H Lu, TW Ling, YT Ko, ML Lee, H Lu, TW Ling, YT Ko, Cleansing data for mining and warehousing, In Database and Expert Systems Applications LNIC, vol. 1677 (Springer, Berlin Heidelberg, 1999). pp. 751-760

20. G Welch, G Bishop, An Introduction to the Kalman Filter (University of North Carolina Press, Chapel Hill, 2006)

21. S Pollin, B Adams, A Bahai, Spatial reuse for practical scenarios: iterative power adjustment from distributed contour estimation and propagation, in The International Conference of Communications (ICC) (IEEE, Piscataway, 2008)

doi:10.1186/1687-1499-2014-72

Cite this article as: Kim and Yeo: Cell coverage estimation by radio

fingerprint data analytics. EURASIP Journal on Wireless Communications and Networking 2014 2014:72.

\section{Submit your manuscript to a SpringerOpen ${ }^{\circ}$ journal and benefit from:}

- Convenient online submission

- Rigorous peer review

- Immediate publication on acceptance

- Open access: articles freely available online

- High visibility within the field

- Retaining the copyright to your article

Submit your next manuscript at $>$ springeropen.com 